PROCEEDINGS OF THE

AMERICAN MATHEMATICAL SOCIETY

Volume 138, Number 1, January 2010, Pages 47-59

S 0002-9939(09)10006-0

Article electronically published on August 20, 2009

\title{
QUIVERS WITH RELATIONS OF HARADA ALGEBRAS
}

\author{
KOTA YAMAURA
}

(Communicated by Birge Huisgen-Zimmermann)

\begin{abstract}
For a finite dimensional algebra $R$, we give an explicit description of quivers with relations of block extensions of $R$. As an application, we describe quivers with relations of Harada algebras by using those of the corresponding quasi-Frobenius algebras.
\end{abstract}

\section{INTRODUCTION}

Two classes of artinian rings have been studied for a long time. One is Nakayama rings, and the other is quasi-Frobenius rings (QF-rings). In the 1980s, Harada introduced the new class of artinian rings, called Harada rings nowadays, which give a common generalization of Nakayama rings and QF-rings. Many authors have studied the structure of Harada rings (e.g. [5, 6, 9, 10, 11, 12, 13, 14]). Among others, Oshiro 14 gave a structure theorem of Harada rings as upper staircase factor rings of block extensions of QF-rings. This plays an important role in the theory of Harada rings.

In [16, Thrall paid attention to three properties of QF-algebras, called QF-1, QF-2, and QF-3. Harada algebras satisfy the property QF-3, which is the condition that the injective hull of the algebra is projective. This property is often called 1-Gorenstein [7, 3] or dominant dimension at least one [15], and often plays an important role in representation theory. Harada algebras give a class of QF-3 algebras, and their indecomposable projective modules have "nice" structure (see Definition 2.2).

In this paper, we study Harada algebras by using the method of representation theory of algebras. In the 1970s, Gabriel introduced quivers with relations in the representation theory of algebras. This gives a powerful tool to calculate modules over algebras (see 2, 4). The aim of this paper is to describe the quivers with relations of Harada algebras by using those of the corresponding QF-algebras. More generally, we give a description of quivers with relations of block extensions of arbitrary finite dimensional algebras.

This paper is organized as follows. In section 2, we recall basic results on Harada algebras, especially their structure theorem in terms of block extensions and upper staircase factor algebras. In section 3, we state our main theorems that describe the quivers with relations of block extensions of arbitrary algebras. We prove them in section 4 . In section 5, we describe quivers with relations of Harada algebras by studying those of upper staircase factor algebras.

Received by the editors May 21, 2008, and, in revised form, April 8, 2009.

2000 Mathematics Subject Classification. Primary 16G10; Secondary 16G70, 18E30.

(C)2009 American Mathematical Society 47 
Throughout this paper, an algebra means a finite dimensional associative algebra over an algebraically closed field $K$. We always deal with right $R$-modules. We denote by $J(M)$ the Jacobson radical of an $R$-module $M$ and by $S(M)$ the socle of $M$. We denote by $\langle x\rangle_{i j}$ the matrix with the $(i, j)$-entry $x$ and other entries 0 .

\section{Block EXTEnsion AND HARAdA ALgEBRAS}

In this section, we recall definitions and basic facts on Harada algebras, in particular block extensions, upper staircase factor algebras, and the structure theorem of Harada algebras. These are due to Oshiro in [12], [13, [14].

We start with giving a definition of a left Harada algebra from its structural point of view.

Definition 2.1. Let $R$ be a basic algebra and $\operatorname{Pi}(R)$ be a complete set of orthogonal primitive idempotents of $R$. We call $R$ a left Harada algebra if $\mathrm{Pi}(R)$ can be arranged such that $\operatorname{Pi}(R)=\left\{e_{i j}\right\}_{i=1}^{m}, n_{i=1}$ where

(1) $e_{i 1} R$ is an injective $R$-module for any $i=1, \cdots, m$,

(2) $e_{i j} R \simeq e_{i, j-1} J(R)$ for any $i=1, \cdots, m, j=2, \cdots, n_{i}$.

In the rest of this section, we recall the structure theorem of Harada algebras. First we have to recall block extensions.

Definition 2.2. Let $R$ be a basic algebra and let $\operatorname{Pi}(R)=\left\{e_{1}, \cdots, e_{m}\right\}$. Then $R$ can be represented as the following matrix form:

$$
R=\left(\begin{array}{cccc}
e_{1} R e_{1} & e_{1} R e_{2} & \cdots & e_{1} R e_{m} \\
e_{2} R e_{1} & e_{2} R e_{2} & \cdots & e_{2} R e_{m} \\
\vdots & \vdots & \ddots & \vdots \\
e_{m} R e_{1} & e_{m} R e_{2} & \cdots & e_{m} R e_{m}
\end{array}\right)
$$

We put $Q_{i}=e_{i} R e_{i}$ and $A_{i j}=e_{i} R e_{j}$. Then $Q_{i}$ is the subalgebra of $R$ with an identity element $e_{i}$, and $A_{i j}$ is a $\left(Q_{i}, Q_{j}\right)$-bimodule.

Let $n_{1}, n_{2}, \cdots, n_{m} \in \mathbb{N}$ be natural numbers. For $1 \leq i, s \leq m, 1 \leq j \leq n_{i}$ and $1 \leq t \leq n_{s}$, we define $P_{i j, s t}$ as follows:

$$
P_{i j, s t}:= \begin{cases}Q_{i} & (i=s, j \leq t) \\ J\left(Q_{i}\right) & (i=s, j>t) \\ A_{i j} & (i \neq s)\end{cases}
$$

and define $n_{i} \times n_{s}$ matrices $P(i, s)$ as

$$
P(i, s):=\left(\begin{array}{cccc}
P_{i 1, s 1} & P_{i 1, s 2} & \cdots & P_{i 1, s n_{s}} \\
P_{i 2, s 1} & P_{i 2, s 2} & \cdots & P_{i 2, s n_{s}} \\
\vdots & \vdots & \ddots & \vdots \\
P_{i n_{i}, s 1} & P_{i n_{i}, s 2} & \cdots & P_{i n_{i}, s n_{s}}
\end{array}\right)=\left\{\begin{array}{ccc}
Q_{i} & \cdots & Q_{i} \\
& \ddots & \vdots \\
J\left(Q_{i}\right) & & Q_{i}
\end{array}\right) \quad(i=s)
$$


We define the block extension $P$ of $R$ for $\left\{n_{1}, \cdots, n_{m}\right\}$ as

$$
P=R\left(n_{1}, \cdots, n_{m}\right)=\left(\begin{array}{cccc}
P(1,1) & P(1,2) & \cdots & P(1, m) \\
P(2,1) & P(2,2) & \cdots & P(2, m) \\
\vdots & \vdots & \ddots & \vdots \\
P(m, 1) & P(m, 2) & \cdots & P(m, m)
\end{array}\right)
$$

Clearly using the usual matrix multiplication, $P$ forms a ring.

We can see that $P$ is a basic finite dimensional $K$-algebra with a complete set of orthogonal primitive idempotents $\left\{f_{i j} \mid 1 \leq i \leq m, 1 \leq j \leq n_{i}\right\}$, where $f_{i j}=\langle 1\rangle_{i j, i j}$. It is known that any block extension of a basic QF-algebra is a basic left Harada algebra (see [12]).

Example 2.3. For example, let $m=2$ and consider the case $n_{1}=3, n_{2}=2$. Then

$$
R(3,2)=\left(\begin{array}{ccc|cc}
Q_{1} & Q_{1} & Q_{1} & A_{12} & A_{12} \\
J\left(Q_{1}\right) & Q_{1} & Q_{1} & A_{12} & A_{12} \\
J\left(Q_{1}\right) & J\left(Q_{1}\right) & Q_{1} & A_{12} & A_{12} \\
\hline A_{21} & A_{21} & A_{21} & Q_{2} & Q_{2} \\
A_{21} & A_{21} & A_{21} & J\left(Q_{2}\right) & Q_{2}
\end{array}\right)
$$

This is a Harada algebra if $R$ is a QF-algebra.

Next we recall upper staircase factor algebras of block extensions.

Definition 2.4. Let $R$ be a basic QF-algebra over a field $K$ with Nakayama permutation $\sigma$ and let $P=R\left(n_{1}, \cdots, n_{m}\right)$ be a block extension in Definition 2.2, Then $P$ is a basic left Harada algebra. First we define a $(P(i, i), P(\sigma(i), \sigma(i)))$-submodule $S(i, \sigma(i))$ of $P(i, \sigma(i))$ as follows.

(I) Case $i=\sigma(i)$. We take a sequence

$$
1 \leq c_{i 1} \leq c_{i 2} \leq \cdots \leq c_{i n_{i}} \leq n_{i}
$$

of natural numbers. We define a subset $S(i, i)$ of $P(i, i)$ as follows:

$$
\begin{gathered}
S_{i j, i t}=\left\{\begin{array}{lll}
0 & \left(1 \leq t \leq c_{i j}\right), \\
S\left(Q_{i}\right) & \left(c_{i j}<t \leq n_{i}\right),
\end{array}\right. \\
P(i, i) \supset S(i, i)=\left(\begin{array}{cccc}
S_{i 1, i 1} & S_{i 1, i 2} & \cdots & S_{i 1, i n_{i}} \\
S_{i 2, i 1} & S_{i 2, i 2} & \cdots & S_{i 2, i n_{i}} \\
\vdots & \vdots & \ddots & \vdots \\
S_{i n_{i}, i 1} & S_{i n_{i}, i 2} & \cdots & S_{i n_{i}, i n_{i}}
\end{array}\right) .
\end{gathered}
$$

We note that $S\left(Q_{i}\right)$ is a left simple and a right simple $Q_{i}$-module. We can see that $S(i, i)$ is a $(P(i, i), P(i, i))$-submodule of $P(i, i)$. It has the following form:

$$
S(i, i)=\left(\begin{array}{l}
0 \cdots 0 \\
0
\end{array}\right)
$$


(II) Case $i \neq \sigma(i)$. We take a sequence

$$
1 \leq c_{i 1} \leq c_{i 2} \leq \cdots \leq c_{i n_{i}} \leq n_{\sigma(i)}
$$

of natural numbers. We define a subset $S(i, \sigma(i))$ of $P(i, \sigma(i))$ as follows:

$$
\begin{gathered}
S_{i j, \sigma(i) t}=\left\{\begin{array}{lll}
0 & \left(1 \leq t \leq c_{i j}\right), \\
S\left(A_{i \sigma(i)}\right) & \left(c_{i j}<t \leq n_{\sigma(i)}\right),
\end{array}\right. \\
P(i, \sigma(i)) \supset S(i, \sigma(i))=\left(\begin{array}{cccc}
S_{i 1, \sigma(i) 1} & S_{i 1, \sigma(i) 2} & \cdots & S_{i 1, \sigma(i) n_{\sigma(i)}} \\
S_{i 2, \sigma(i) 1} & S_{i 2, \sigma(i) 2} & \cdots & S_{i 2, \sigma(i) n_{\sigma(i)}} \\
\vdots & \vdots & \ddots & \vdots \\
S_{i n_{i}, \sigma(i) 1} & S_{i n_{i}, \sigma(i) 2} & \cdots & S_{i n_{i}, \sigma(i) n_{\sigma(i)}}
\end{array}\right) .
\end{gathered}
$$

We note that $S\left(A_{i \sigma(i)}\right)$ is a left simple $Q_{i}$-module and a right simple $Q_{\sigma(i)^{-}}$ module. We can see that $S(i, \sigma(i))$ is a $(P(i, i), P(\sigma(i), \sigma(i)))$-submodule of $P(i, \sigma(i))$. It has the following form:

$$
S(i, \sigma(i))=\left(\begin{array}{lll}
0 \cdots 0 & S\left(A_{i \sigma i}\right) \\
&
\end{array}\right.
$$

Next we define a subset $X$ of $P$ by putting

$$
\begin{gathered}
P(i, s) \supset X(i, s)=\left\{\begin{array}{lll}
S(i, \sigma(i)) & (s=\sigma(i)), \\
0 & & (s \neq \sigma(i)),
\end{array}\right. \\
X=\left(\begin{array}{cccc}
X(1,1) & X(1,2) & \cdots & X(1, m) \\
X(2,1) & X(2,2) & \cdots & X(2, m) \\
\vdots & \vdots & \ddots & \vdots \\
X(m, 1) & X(m, 2) & \cdots & X(m, m)
\end{array}\right) .
\end{gathered}
$$

Then $X$ is an ideal of $P$. We define an upper staircase factor algebra $\bar{P}$ of $P$ by

$$
\bar{P}=P / X=R\left(n_{1}, \cdots, n_{m}\right) / X .
$$

It is known that any upper staircase factor algebra of a block extension of a basic QF-algebra is a basic left Harada algebra (see [14]). Conversely all left Harada algebras are constructed by these operations. In fact, the following well-known result holds.

Theorem 2.5 (Structure Theorem of Harada algebras [14]). For any basic left Harada algebra $T$, there exists a basic QF-algebra $R$ such that $T$ is isomorphic to an upper staircase factor algebra of a block extension of $R$.

We call the above $R$ the frame $Q F$-algebra of $T$.

The above theorem motivates us to consider the following question:

Question. Is the quiver with relations of a Harada algebra described by using those of the frame QF-algebra?

The answer is "yes". We describe the quivers with relations of block extensions of arbitrary (not necessarily QF) algebras $R$ by using those of $R$ in sections 3 and 
4. Moreover for the case that $R$ is $\mathrm{QF}$, we describe the quivers with relations of upper staircase factor algebras in section 5 .

Example 2.6. We consider $P=R(3,2)$ of Example 2.3. We assume that $R$ is a basic QF-algebra with Nakayama permutation identity. For example, we take the following sequence:

$$
c_{11}=1, c_{12}=2, c_{13}=2, c_{21}=1, c_{22}=2 .
$$

Then $X$ defined above is as follows:

$$
X=\left(\begin{array}{ccc|cc}
0 & S\left(Q_{1}\right) & S\left(Q_{1}\right) & 0 & 0 \\
0 & 0 & S\left(Q_{1}\right) & 0 & 0 \\
0 & 0 & S\left(Q_{1}\right) & 0 & 0 \\
\hline 0 & 0 & 0 & 0 & S\left(Q_{2}\right) \\
0 & 0 & 0 & 0 & 0
\end{array}\right) .
$$

The factor algebra $P / X$ is a left Harada algebra.

\section{MAIN THEOREM}

In this section, we first recall basic definitions on presentations of algebras by using quivers with relations (we refer to [2, Chapter II] for the details). Next we state the main result of this paper, which describes quivers with relations of block extensions of algebras $R$ by using those of $R$.

Let $R$ be a basic finite dimensional algebra over an algebraically closed field $K$. We use the notation in Definition 2.2. The Jacobson radical of $R$ is given by

$$
J(R)=\left(\begin{array}{cccc}
J\left(Q_{1}\right) & A_{12} & \cdots & A_{1 m} \\
A_{21} & J\left(Q_{2}\right) & \cdots & A_{2 m} \\
\vdots & \vdots & \ddots & \vdots \\
A_{m 1} & A_{m 2} & \cdots & J\left(Q_{m}\right)
\end{array}\right)
$$

(see [1]). Hence we have

$$
J(R)^{2}=\left(\begin{array}{ccc}
X_{11} & \cdots & X_{1 m} \\
\vdots & & \vdots \\
X_{m 1} & \cdots & X_{m m}
\end{array}\right)
$$

where

$$
X_{i j}= \begin{cases}J\left(Q_{i}\right)^{2}+\sum_{k \neq i} A_{i k} A_{k i} & (i=j), \\ J\left(Q_{i}\right) A_{i j}+A_{i j} J\left(Q_{j}\right)+\sum_{k \neq i, j} A_{i k} A_{k j} & (i \neq j) .\end{cases}
$$

We put

$$
d_{i j}= \begin{cases}\operatorname{dim}_{K} J\left(Q_{i}\right) / X_{i i} & (i=j), \\ \operatorname{dim}_{K} A_{i j} / X_{i j} & (i \neq j) .\end{cases}
$$

Then the quiver $Q$ of $R$ is defined as follows:

(a) The set of vertices of $Q$ is $\{1,2, \cdots, m\}$.

(b) There are $d_{i j}$ arrows $\left\{\alpha_{i j}^{1}, \alpha_{i j}^{2}, \cdots, \alpha_{i j}^{d_{i j}}\right\}$ from $i$ to $j$.

We define the path algebra of $Q$. Let $K Q$ be a $K$-vector space with the basis consisting of all paths in $Q$. We define multiplication of $K Q$ as follows: For any 
paths $\alpha=\alpha_{1} \cdots \alpha_{s}, \beta=\beta_{1} \cdots \beta_{t}\left(\alpha_{k}, \beta_{l}\right.$ are arrows), we let $i$ be the end vertex of $\alpha_{k}$ and let $j$ be the starting vertex of $\beta_{1}$. Then we define

$$
\alpha \beta= \begin{cases}\alpha_{1} \cdots \alpha_{s} \beta_{1} \cdots \beta_{t} & (i=j), \\ 0 & (i \neq j) .\end{cases}
$$

We extend the multiplication linearly to $K Q$. Then we have a $K$-algebra $K Q$ called the path algebra of $Q$. We call $p \in K Q$ basic if all paths appearing in $p$ have the same source and target.

We fix a basis $\left\{x_{i j}^{1}, x_{i j}^{2}, \cdots, x_{i j}^{d_{i j}}\right\}$ of $K$-vector spaces $J\left(Q_{i}\right) / X_{i i}$ and $A_{i j} / X_{i j}$. By the universal property of path algebras, we can define a $K$-algebra homomorphism $\varphi: K Q \longrightarrow R$ by

$$
\begin{aligned}
K Q \ni i & \longmapsto\langle 1\rangle_{i i} \in R, \\
K Q \ni \alpha_{i j}^{t} & \longmapsto\left\langle x_{i j}^{t}\right\rangle_{i j} \in R .
\end{aligned}
$$

Then $\varphi$ is surjective. Consequently, we have a $K$-algebra isomorphism,

$$
K Q / \operatorname{Ker} \varphi \simeq R
$$

We fix a set of generators $\left\{\rho_{1}, \cdots, \rho_{w}\right\}$ of the two-sided ideal $\operatorname{Ker} \varphi$ of $R$. We can assume that $\rho_{i}$ is basic for any $i$.

Now we state our main results, which give a quiver with relations of a block extension algebra of $R$.

Theorem 3.1. Let $n_{1}, \cdots, n_{m} \in \mathbb{N}$ and put $P=R\left(n_{1}, \cdots, n_{m}\right)$. The quiver $Q^{\prime}$ of $P$ is given as follows:

(a) The set of vertices of $Q^{\prime}$ is $\left\{(i, j) \mid 1 \leq i \leq m, 1 \leq j \leq n_{i}\right\}$.

(b) We have the following two kinds of arrows:

- An arrow $\delta_{i j}$ from $(i, j)$ to $(i, j+1)$.

- $d_{i s}$ arrows $\left\{\beta_{i s}^{1}, \beta_{i s}^{2}, \cdots, \beta_{i s}^{d_{i s}}\right\}$ from $\left(i, n_{i}\right)$ to $(s, 1)$.

To describe relations for $P$, we have to define the extension map.

Definition 3.2. Let $K Q_{+}$(respectively, $K Q_{+}^{\prime}$ ) be a $K$-subspace of $K Q$ (respectively, $\left.K Q^{\prime}\right)$ generated by all paths of length $\geq 1$. Using $\delta_{i j}$ defined in Theorem 3.1. we put

$$
\delta_{i}=\delta_{i 1} \delta_{i 2} \cdots \delta_{i n_{i}-1} \in K Q^{\prime} .
$$

We define a $K$-linear map $e: K Q_{+} \longrightarrow K Q_{+}^{\prime}$, called the extension map, as follows: Any path

$$
p=\alpha_{i_{1} i_{2}}^{t_{1}} \alpha_{i_{2} i_{3}}^{t_{2}} \cdots \alpha_{i_{k} i_{k+1}}^{t_{k}}
$$

of $K Q$ corresponds to the path

$$
e(p)=\beta_{i_{1} i_{2}}^{t_{1}} \delta_{i_{2}} \beta_{i_{2} i_{3}}^{t_{2}} \cdots \beta_{i_{k-1} i_{k}}^{t_{k-1}} \delta_{i_{k}} \beta_{i_{k} i_{k+1}}^{t_{k}}
$$

of $K Q^{\prime}$. Clearly $e$ is an injection.

Then we can describe relations for $P$.

Theorem 3.3. Under the hypotheses of Theorem 3.1, we have a K-algebra isomorphism,

$$
P \simeq K Q^{\prime} /\left(e\left(\rho_{1}\right), \cdots, e\left(\rho_{w}\right)\right)
$$


Example 3.4. Let $R$ be a $K$-algebra given by the following quiver with relations:

$$
\alpha_{11} \bigcirc 1 \underset{\alpha_{21}}{\stackrel{\alpha_{12}}{\rightleftarrows} 2}\left\{\begin{array}{l}
\alpha_{11}^{3}=\alpha_{12} \alpha_{21}, \\
\alpha_{11} \alpha_{12}=0 \\
\alpha_{21} \alpha_{11}=0
\end{array}\right.
$$

Then $R$ can be represented as the following matrix form:

$$
R=\left(\begin{array}{cc}
K\left[\alpha_{11}\right] /\left(\alpha_{11}\right)^{4} & K \alpha_{12} \\
K \alpha_{21} & K\left[\alpha_{21} \alpha_{12}\right] /\left(\alpha_{21} \alpha_{12}\right)^{2}
\end{array}\right) .
$$

For example, $R(3,2)$ can be represented as the matrix form

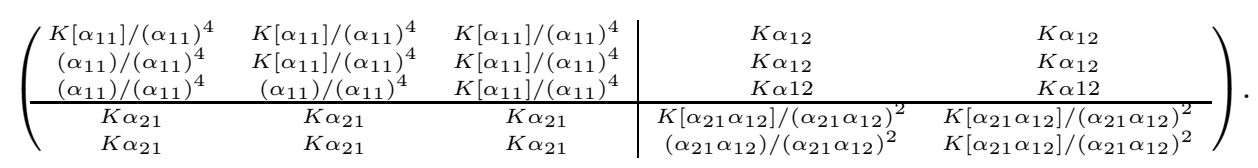

The quiver with relations of $R(3,2)$ is given by the following:

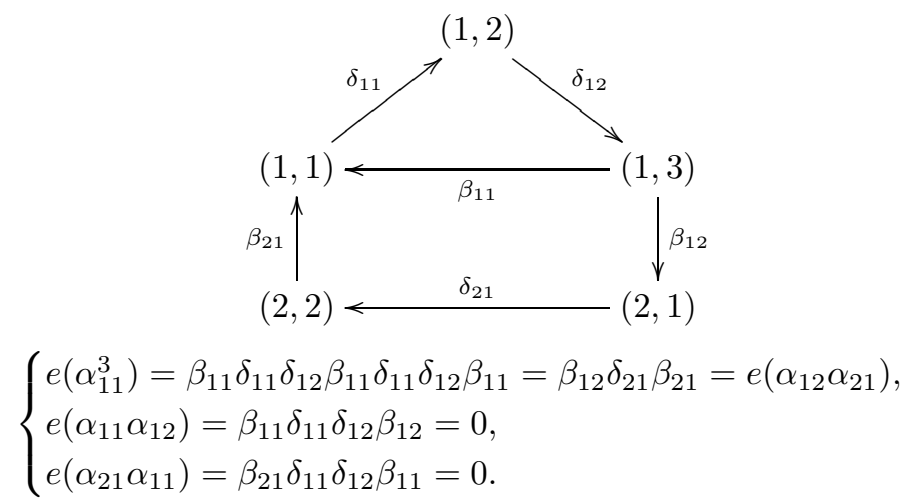

\section{Proof of MAin theorem}

In this section, we keep the notation of the previous section. We prove Theorems 3.1 and 3.3 .

Let $P$ be a block extension $R\left(n_{1}, \cdots, n_{m}\right)$. The Jacobson radical of $P$ is given by

$$
\begin{gathered}
J(P)=\left(\begin{array}{cccc}
J(1,1) & J(1,2) & \cdots & J(1, m) \\
J(2,1) & J(2,2) & \cdots & J(2, m) \\
\vdots & \vdots & \ddots & \vdots \\
J(m, 1) & J(m, 2) & \cdots & J(m, m)
\end{array}\right), \\
P_{i j, s t} \supset J_{i j, s t}=\left\{\begin{array}{lll}
Q_{i} & (i=s, j+1 \leq t), \\
J\left(Q_{i}\right) & (i=s, j+1>t), \\
A_{i s} & (i \neq s),
\end{array}\right.
\end{gathered}
$$


where we use the notation in Definition 2.2, Then

$$
\begin{aligned}
P(i, s) \supset J(i, s) & =\left(\begin{array}{ccc}
J_{i 1, s 1} & \cdots & J_{i 1, s n_{s}} \\
\vdots & & \vdots \\
J_{i n_{i}, s 1} & \cdots & J_{i n_{i}, s n_{s}}
\end{array}\right) \\
& =\left\{\begin{array}{ccccc}
J\left(Q_{i}\right) & Q_{i} & \cdots & \cdots & Q_{i} \\
& J\left(Q_{i}\right) & Q_{i} & \cdots & Q_{i} \\
& & \ddots & \ddots & \vdots \\
& & & J\left(Q_{i}\right) & Q_{i} \\
J\left(Q_{i}\right) & & & & J\left(Q_{i}\right)
\end{array}\right) \quad(i=s), \\
\left(\begin{array}{ccc}
A_{i s} & \cdots & A_{i s} \\
\vdots & & \vdots \\
A_{i s} & \cdots & A_{i s}
\end{array}\right) & (i \neq s) .
\end{aligned}
$$

Now let us calculate $J(P)^{2}$. We denote by $Y_{i j, s t}$ the $(i j, s t)$-entry of $J(P)^{2}$.

(I) Case $i=s$.

$$
\begin{aligned}
Y_{i j, i t} & =\sum_{k=1}^{m} \sum_{l=1}^{n_{k}} J_{i j, k l} J_{k l, i t} \\
& =\sum_{l=1}^{n_{i}} J_{i j, i l} J_{i l, i t}+\sum_{k=1, k \neq i}^{m} \sum_{l=1}^{n_{k}} J_{i j, k l} J_{k l, i t} \\
& = \begin{cases}Q_{i} & (j+1<t), \\
J\left(Q_{i}\right) & \left(j+1 \geq t,(j, t) \neq\left(n_{i}, 1\right)\right), \\
J\left(Q_{i}\right)^{2}+\sum_{k=1, k \neq i}^{m} A_{i k} A_{k i}=X_{i i} & \left((j, t)=\left(n_{i}, 1\right)\right),\end{cases}
\end{aligned}
$$

where $X_{i i}$ is in (3.1).

(II) Case $i \neq s$.

$$
\begin{aligned}
Y_{i j, s t} & =\sum_{k=1}^{m} \sum_{l=1}^{n_{k}} J_{i j, k l} J_{k l, s t} \\
& =\sum_{l=1}^{n_{i}} J_{i j, i l} J_{i l, s t}+\sum_{l=1}^{n_{j}} J_{i j, j l} J_{j l, s t}+\sum_{k=1, k \neq i, j}^{m} \sum_{l=1}^{n_{k}} J_{i j, k l} J_{k l, s t} \\
& = \begin{cases}A_{i s} & \left((j, t) \neq\left(n_{i}, 1\right)\right), \\
J\left(Q_{i}\right) A_{i s}+A_{i s} J\left(Q_{s}\right)+\sum_{k=1, k \neq i, j}^{m} A_{i k} A_{k s}=X_{i s} & \left((j, t)=\left(n_{i}, 1\right)\right),\end{cases}
\end{aligned}
$$

where $X_{i s}$ is in (3.1). 
Therefore

$$
\begin{aligned}
& P(i, s) \supset Y(i, s)=\left(\begin{array}{ccc}
Y_{i 1, s 1} & \cdots & Y_{i 1, s n_{s}} \\
\vdots & & \vdots \\
Y_{i n_{i}, s 1} & \cdots & Y_{i n_{i}, s n_{s}}
\end{array}\right) \\
& =\left\{\begin{array}{ccccc}
J\left(Q_{i}\right) & J\left(Q_{i}\right) & & & Q_{i} \\
\vdots & J\left(Q_{i}\right) & J\left(Q_{i}\right) & & \\
\vdots & & \ddots & \ddots & \\
J\left(Q_{i}\right) & J\left(Q_{i}\right) & & J\left(Q_{i}\right) & J\left(Q_{i}\right) \\
X_{i i} & J\left(Q_{i}\right) & \ldots & \ldots & J\left(Q_{i}\right)
\end{array}\right) \quad(i=s), \\
& J(P)^{2}=\left(\begin{array}{cccc}
Y(1,1) & Y(1,2) & \cdots & Y(1, m) \\
Y(2,1) & Y(2,2) & \cdots & Y(2, m) \\
\vdots & \vdots & \ddots & \vdots \\
Y(m, 1) & Y(m, 2) & \cdots & Y(m, m)
\end{array}\right) .
\end{aligned}
$$

Thus we have

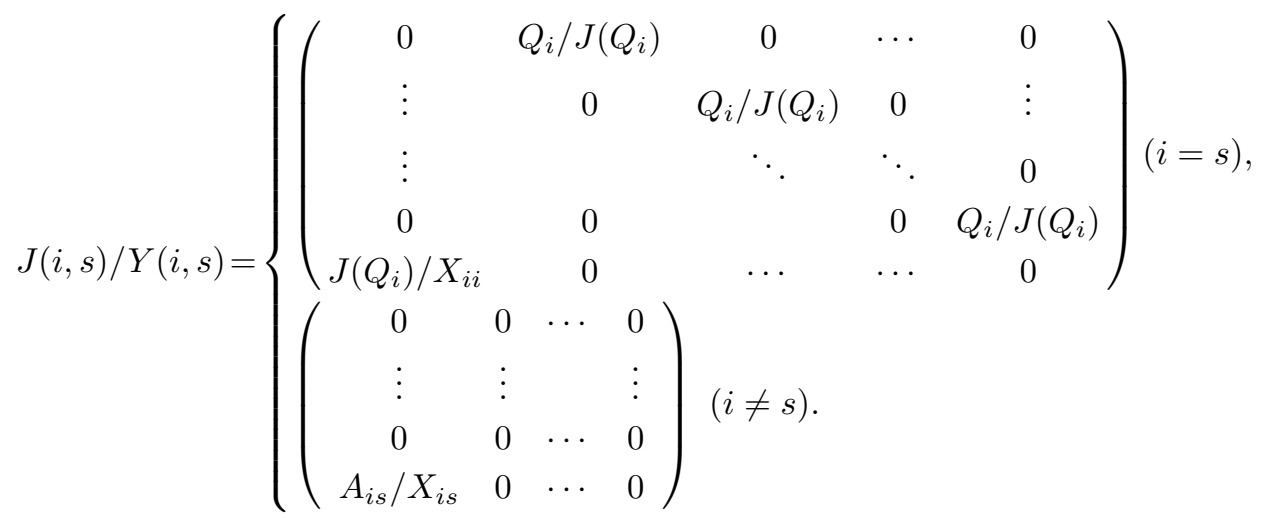

Consequently the quiver $Q^{\prime}$ of $P$ is given by Theorem 3.1 .

Next, we shall prove Theorem 3.3. Define a $K$-algebra homomorphism $\varphi^{\prime}$ : $K Q^{\prime} \longrightarrow P$ by

$$
\begin{aligned}
K Q^{\prime} \ni(i, j) & \longmapsto\langle 1\rangle_{i j, i j} \in P, \\
K Q^{\prime} \ni \delta_{i j} & \longmapsto\langle 1\rangle_{i j, i j+1} \in P, \\
K Q^{\prime} \ni \beta_{i s}^{t} & \longmapsto\left\langle x_{i s}^{t}\right\rangle_{i n_{i}, s 1} \in P .
\end{aligned}
$$

Then $\varphi^{\prime}$ is surjective. Consequently, we have a $K$-algebra isomorphism,

$$
K Q^{\prime} / \operatorname{Ker} \varphi^{\prime} \simeq P \text {. }
$$

We only have to show that the two-sided ideal $\operatorname{Ker} \varphi^{\prime}$ of $P$ is generated by the $e\left(\rho_{1}\right), \cdots, e\left(\rho_{w}\right)$. Let us start with giving elementary properties of the extension map. 
Lemma 4.1. For the extension map e: $K Q_{+} \longrightarrow K Q_{+}^{\prime}$, the following hold:

(1) The two-sided ideal $\operatorname{Ker} \varphi^{\prime}$ of $P$ is generated by elements in $\operatorname{Im} e$.

(2) For any $p \in K Q_{+}, \varphi(p)=0$ if and only if $\varphi^{\prime}(e(p))=0$.

(3) For any path $p \in K Q_{+}$ending at $j$ and any path $q \in K Q_{+}$starting at $j$, we have

$$
e(p q)=e(p) \delta_{j} e(q)
$$

where $\delta_{j}$ is defined in Definition 3.2 .

Proof. (1) We take $p^{\prime} \in \operatorname{Ker} \varphi^{\prime}$. We can assume that $p^{\prime}$ is basic. By the definition of the quiver $Q^{\prime}$, we can write $p^{\prime}$ as follows:

$$
p^{\prime}=\delta_{i j} \cdots \delta_{i n_{i}} p^{\prime \prime} \delta_{s 1} \cdots \delta_{s t} \quad\left(p^{\prime \prime} \in \operatorname{Im} e\right) .
$$

We have

$$
\begin{aligned}
\varphi^{\prime}\left(p^{\prime}\right) & =\varphi^{\prime}\left(\delta_{i j} \cdots \delta_{i n_{i}} p^{\prime \prime} \delta_{s 1} \cdots \delta_{s t}\right) \\
& =\varphi^{\prime}\left(\delta_{i j}\right) \cdots \varphi^{\prime}\left(\delta_{i n_{i}}\right) \varphi^{\prime}\left(p^{\prime \prime}\right) \varphi^{\prime}\left(\delta_{s 1}\right) \cdots \varphi^{\prime}\left(\delta_{s t}\right) \\
& =\langle 1\rangle_{i j, i j+1} \cdots\langle 1\rangle_{i n_{i}-1, i n_{i}} \varphi^{\prime}\left(p^{\prime \prime}\right)\langle 1\rangle_{s 1, s 2} \cdots\langle 1\rangle_{s t, s t+1} .
\end{aligned}
$$

We have $\varphi^{\prime}\left(p^{\prime \prime}\right)=0$ since $\varphi^{\prime}\left(p^{\prime}\right)=0$. Consequently, $p^{\prime \prime} \in \operatorname{Ker} \varphi^{\prime} \cap \operatorname{Im} e$. Thus the assertion follows.

(2) We take $K Q_{+} \ni p=\alpha_{i_{1} i_{2}}^{t_{1}} \alpha_{i_{2} i_{3}}^{t_{2}} \cdots \alpha_{i_{k} i_{k+1}}^{t_{k}}$. Then we have

$$
\begin{aligned}
\varphi(p) & =\left\langle x_{i_{1} i_{2}}^{t_{1}}\right\rangle_{i_{1} i_{2}}\left\langle x_{i_{2} i_{3}}^{t_{2}}\right\rangle_{i_{2} i_{3}} \cdots\left\langle x_{i_{k} i_{k+1}}^{t_{k}}\right\rangle_{i_{k} i_{k+1}} \\
& =\left\langle x_{i_{1} i_{2}}^{t_{1}} x_{i_{2} i_{3}}^{t_{2}} \cdots x_{i_{k} i_{k+1}}^{t_{k}}\right\rangle_{i_{1} i_{k+1}} .
\end{aligned}
$$

On the other hand, we have

$$
\begin{aligned}
\varphi^{\prime}(e(p)) & =\varphi^{\prime}\left(\beta_{i_{1} i_{2}}^{t_{1}} \delta_{i_{2}} \beta_{i_{2} i_{3}}^{t_{2}} \cdots \beta_{i_{k} i_{k+1}}^{t_{k}}\right) \\
& =\varphi^{\prime}\left(\beta_{i_{1} i_{2}}^{t_{1}}\right) \varphi^{\prime}\left(\delta_{i_{2}}\right) \varphi^{\prime}\left(\beta_{i_{2} i_{3}}^{t_{2}}\right) \cdots \varphi^{\prime}\left(\beta_{i_{k} i_{k+1}}^{t_{k}}\right) \\
& =\left\langle x_{i_{1} i_{2}}^{t_{1}}\right\rangle_{i_{1} n_{i_{1}}, i_{2} 1}\langle 1\rangle_{i_{2} 1, i_{2} n_{i_{2}}}\left\langle x_{i_{2} i_{3}}^{t_{2}}\right\rangle_{i_{2} n_{i_{2}}, i_{3} 1} \cdots\left\langle x_{i_{k} i_{k+1}}^{t_{k}}\right\rangle_{i_{k} n_{i_{k}}, i_{k+1} 1} \\
& =\left\langle x_{i_{1} i_{2}}^{t_{1}} x_{i_{2} i_{3}}^{t_{2}} \cdots x_{i_{k} i_{k+1}}^{t_{k}}\right\rangle_{i_{1} n_{i_{1}}, i_{k+1} 1} .
\end{aligned}
$$

Consequently, for any basic element $p \in K Q_{+}$from $i$ to $j$, there exists $r \in e_{i} R e_{j}$ such that

$$
\begin{aligned}
\varphi(p) & =\langle r\rangle_{i j}, \\
\varphi^{\prime}(e(p)) & =\langle r\rangle_{i n_{i}, j 1} .
\end{aligned}
$$

Thus $\varphi(p)=0$ if and only if $r=0$ and if and only if $\varphi^{\prime}(e(p))=0$.

(3) This follows from the definition of $e$.

Now we complete the proof of Theorem 3.3. Put $I^{\prime}=\left(e\left(\rho_{1}\right), \cdots, e\left(\rho_{w}\right)\right)$. We shall show $I^{\prime}=\operatorname{Ker} \varphi^{\prime}$. We have $I^{\prime} \subset \operatorname{Ker} \varphi^{\prime}$ by Lemma 4.1 (2).

By Lemma 4.1 (1), we only have to show $\operatorname{Ker} \varphi^{\prime} \cap \operatorname{Im} e \subset I^{\prime}$. Assume that $p \in K Q_{+}$satisfies $e(p) \in \operatorname{Ker} \varphi^{\prime}$. By Lemma 4.1 (2), we have $p \in \operatorname{Ker} \varphi$. We can write

We have

$$
p=a_{1} \rho_{1} b_{1}+a_{2} \rho_{2} b_{2}+\cdots+a_{w} \rho_{w} b_{w} \quad\left(a_{i}, b_{i} \in K Q\right) .
$$

$$
e(p)=e\left(a_{1} \rho_{1} b_{1}\right)+e\left(a_{2} \rho_{2} b_{2}\right)+\cdots+e\left(a_{w} \rho_{w} b_{w}\right) .
$$

By Lemma 4.1 (3), each $e\left(a_{i} \rho_{i} b_{i}\right)$ is contained in $I^{\prime}$. Thus $p^{\prime} \in I^{\prime}$. Consequently $I^{\prime}=\operatorname{Ker} \varphi^{\prime}$. 


\section{Application of main theorem to Harada algebras}

In this section, we describe quivers with relations of upper staircase factor algebras. Then we can describe quivers with relations of Harada algebras completely. We use the notation which was used in previous sections.

We assume that $R$ is a basic QF-algebra with Nakayama permutation $\sigma$. We put $P=R\left(n_{1}, \cdots, n_{m}\right)$ (see Definition 2.2). For $1 \leq i \leq m$, we take a sequence

$$
1 \leq c_{i 1} \leq c_{i 2} \leq \cdots \leq c_{i n_{i}} \leq n_{i} .
$$

Then we consider an upper staircase factor algebra $\bar{P}=P / X$, where $X$ is defined by the above sequences (see Definition 2.4). For $1 \leq i \leq m$, we define $l_{i 0}, l_{i 1}, \cdots, l_{i u_{i}}$ to satisfy the following conditions:

(1) $l_{i 0}=0$ and $l_{i u_{i}}=n_{i}$.

(2) $c_{i, l_{i j-1}+1}=\cdots=c_{i l_{i j}}$ for $1 \leq j \leq u_{i}$.

(3) $c_{i l_{i j}}<c_{i, l_{i j}+1}$ for $1 \leq j \leq u_{i}$.

Now let us calculate relations for $\bar{P}$. We take a path $\theta_{i}$ in $Q$ from $i$ to $\sigma(i)$ such that $\varphi\left(\theta_{i}\right) \in S\left(e_{i} R\right)$. We put

$$
\theta_{i}^{\prime}=\delta_{i} e\left(\theta_{i}\right) \delta_{\sigma(i)}=\delta_{i 1} \cdots \delta_{i, n_{i}-1} e\left(\theta_{i}\right) \delta_{\sigma(i) 1} \cdots \delta_{\sigma(i), n_{\sigma(i)}-1}
$$

for $1 \leq i \leq m$ (see Definition 3.2 for $\delta_{i}$ and $e$ ). Then we have the following lemma.

Lemma 5.1. For $1 \leq i \leq m, \varphi^{\prime}\left(\theta_{i}^{\prime}\right) \in S\left(f_{i 1} P\right)$.

Proof. By the proofs of Lemma 4.1 (1) and Lemma 4.1 (2), we have $\varphi^{\prime}\left(\theta_{i}^{\prime}\right) \neq 0$. Clearly $\varphi^{\prime}\left(\theta_{i}^{\prime}\right) \in f_{i 1} P$. To show $\varphi^{\prime}\left(\theta_{i}^{\prime}\right) \in S\left(f_{i 1} P\right)$, we only have to show that $\varphi^{\prime}\left(\theta_{i}^{\prime}\right) J(P)=0$. For any $1 \leq t \leq d_{\sigma(i) s}$, we have by definition of the extension map,

$$
\theta_{i}^{\prime} \beta_{\delta(i) s}^{t}=\delta_{i} e(\theta) \delta_{\sigma(i)} \beta_{\delta(i) s}^{t}=\delta_{i} e\left(\theta_{i} \alpha_{\sigma(i) s}^{t}\right) .
$$

Since $\varphi\left(\theta_{i} \alpha_{\sigma(i) s}^{t}\right)=0$ and by Lemma $4.1(2)$, we have $\varphi^{\prime}\left(e\left(\theta_{i} \alpha_{\sigma(i) s}^{t}\right)\right)=0$. Consequently $\varphi^{\prime}\left(\theta_{i}^{\prime}\right) \varphi^{\prime}\left(\beta_{\delta(i) s}^{t}\right)=\varphi^{\prime}\left(\theta_{i}^{\prime} \beta_{\delta(i) s}^{t}\right)=0$. We have $\varphi^{\prime}\left(\theta_{i}^{\prime}\right) J(P)=0$.

We put

$$
\theta_{i}^{\prime}(u, v)= \begin{cases}\delta_{i u} \cdots \delta_{i n_{i}-1} e\left(\theta_{i}\right) \delta_{\sigma(i) 1} \cdots \delta_{\sigma(i) v} & \left(1 \leq u \leq n_{i}-1,1 \leq v \leq n_{\sigma(i)}-1\right) \\ e\left(\theta_{i}\right) \delta_{\sigma(i) 1} \cdots \delta_{\sigma(i) v} & \left(u=n_{i}, 1 \leq v \leq n_{\sigma(i)}-1\right) .\end{cases}
$$

Then the two-sided ideal $X$ of $P$ is generated by

$$
\varphi^{\prime}\left(\theta_{i}^{\prime}\left(l_{i j}, c_{i l_{i j}}\right)\right) \quad\left(1 \leq i \leq m, 1 \leq j \leq u_{i}\right),
$$

since we can obtain any entry in $X(i, \sigma(i))$ by multiplying $\varphi^{\prime}(\delta)$ 's to one of these elements. Consequently we have the following theorem.

Theorem 5.2. Under the hypotheses as above, we have a K-algebra isomorphism

$$
\bar{P} \simeq K Q^{\prime} / I^{\prime}
$$

for an ideal

$$
I^{\prime}=\left(e\left(\rho_{1}\right), \cdots, e\left(\rho_{w}\right)\right)+\left(\theta_{i}^{\prime}\left(l_{i j}, c_{i l_{i j}}\right) \mid 1 \leq i \leq m, 1 \leq j \leq u_{i}\right)
$$

of $K Q^{\prime}$. 
Example 5.3. We consider $P=R(3,2)$ in Example 3.4. The Nakayama permutation of $R$ is the identity. We use the ideal $X$ in Example 2.6.

$$
X=\left(\begin{array}{ccc|cc}
0 & \left(\alpha_{11}\right)^{3} /\left(\alpha_{11}\right)^{4} & \left(\alpha_{11}\right)^{3} /\left(\alpha_{11}\right)^{4} & 0 & 0 \\
0 & 0 & \left(\alpha_{11}\right)^{3} /\left(\alpha_{11}\right)^{4} & 0 & 0 \\
0 & 0 & \left(\alpha_{11}\right)^{3} /\left(\alpha_{11}\right)^{4} & 0 & 0 \\
\hline 0 & 0 & 0 & 0 & \left(\alpha_{21} \alpha_{12}\right) /\left(\alpha_{21} \alpha_{12}\right)^{2} \\
0 & 0 & 0 & 0 & 0
\end{array}\right)
$$

In this case, $l_{11}=1, l_{12}=3, l_{21}=1, l_{22}=2$. Then the quiver with relations of the upper staircase factor algebra $P / X$ is given by those in Example 3.4 with additional relations

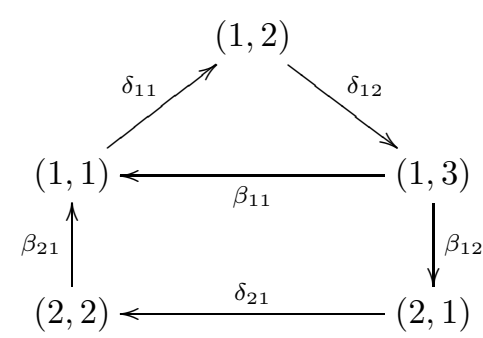

$$
\left\{\begin{array}{l}
\delta_{11} \delta_{12} e\left(\alpha_{11}^{3}\right) \delta_{11}=0 \\
e\left(\alpha_{11}^{3}\right) \delta_{11} \delta_{12}=0 \\
\delta_{21} e\left(\alpha_{21} \alpha_{12}\right) \delta 21=0
\end{array}\right.
$$

where $e\left(\alpha_{11}^{3}\right)=\beta_{11} \delta_{11} \delta_{12} \beta_{11} \delta_{11} \delta_{12} \beta_{11}$ and $e\left(\alpha_{21} \alpha_{12}\right)=\beta_{12} \delta_{11} \delta_{12} \beta_{12}$.

\section{REFERENCES}

[1] F. W. Anderson, K. R. Fuller: Rings and Categories of Modules (second edition), Graduate Texts in Math., 13, Springer-Verlag, Heidelberg-New York-Berlin (1992). MR1245487 (94i:16001)

[2] I. Assem, D. Simson, A. Skowroński: Elements of the Representation Theory of Associative Algebras, London Mathematical Society Student Texts, 65, Cambridge University Press (2006). MR2197389 (2006j:16020)

[3] M. Auslander, I. Reiten: $k$-Gorenstein algebras and syzygy modules, J. Pure Appl. Algebra 92 (1994), 1-27. MR1259667 (95d:16008)

[4] M. Auslander, I. Reiten, S. Smalø: Representation Theory of Artin Algebras, Cambridge Studies in Advanced Mathematics, 36, Cambridge University Press (1995). MR.1314422 (96c:16015)

[5] Y. Baba, K. Iwase: On quasi-Harada rings, J. Algebra 185 (1996), 544-570. MR1417385 (98a:16026)

[6] Y. Baba, K. Oshiro: Classical Artinian Rings and Related Topics, preprint.

[7] R. Fossum, P. Griffith, I. Reiten: Trivial Extensions of Abelian Categories, Lecture Notes in Mathematics, Vol. 456, Springer-Verlag, Berlin-New York (1975). MR0389981 (52:10810)

[8] M. Harada: Nonsmall modules and noncosmall modules, Ring Theory. Proceedings of 1978 Antwerp Conference, Dekker, New York (1979), 669-690. MR0563315 (81c:16022)

[9] K. Koike: Almost self-duality and Harada rings, J. Algebra 254 (2002), 336-361. MR.1933873 (2004a:16006)

[10] K. Oshiro: Lifting modules, extending modules and their applications to QF-rings, Hokkaido Math. J. 13 (1984), 310-338. MR764267 (86b:16008a)

[11] K. Oshiro: Lifting modules, extending modules and their applications to generalized uniserial rings, Hokkaido Math. J. 13 (1984), 339-346. MR764268 (86b:16008b)

[12] K. Oshiro: On Harada rings. I, Math. J. Okayama Univ. 31 (1989), 161-178. MR.1043359 (91f:16025)

[13] K. Oshiro: On Harada rings. II, Math. J. Okayama Univ. 31 (1989), 179-188. MR.1043359 (91f:16025)

[14] K. Oshiro: On Harada rings. III, Math. J. Okayama Univ. 32 (1990), 111-118. MR.1112019 (92k:16027) 
[15] H. Tachikawa: Quasi-Frobenius rings and generalizations. QF -3 and QF -1 rings, Lecture Notes in Mathematics, Vol. 351, Springer-Verlag, Berlin-New York (1973). MR0349740 $(50: 2233)$

[16] R. M. Thrall: Some generalization of quasi-Frobenius algebras, Trans. Amer. Math. Soc. 64 (1948), 173-183. MR0026048 (10:98c)

Graduate School of Mathematics, Nagoya University, Frocho, Chikusaku, Nagoya, 464-8602, JAPAN

E-mail address: m07052d@math.nagoya-u.ac.jp 